\title{
Hypersensitivity Pneumonitis Associated with Inhalation of Catechin-Rich Green Tea Extracts
}

\author{
Hiroshi Otera $^{a}$ Kimihide Tada $^{a}$ Toshiyasu Sakurai ${ }^{a} \quad$ Kimio Hashimoto $^{\mathrm{b}}$ Akihiko Ikeda $^{\mathrm{a}}$ \\ Departments of a Respiratory Medicine and ${ }^{b}$ Pathology, Nishi-Kobe Medical Center, Kobe, Japan
}

\section{Established Facts}

- Green tea possesses many useful properties without severe side effects.

\section{Novel Insights}

- Catechin powder inhalation can trigger hypersensitivity pneumonitis.

\section{Key Words}

Hypersensitivity pneumonitis · Catechin • Green tea

\section{Abstract}

A 51-year-old man presented with fever and fatigue after 3.5 months of antituberculosis therapy. High-resolution computed tomography of his chest revealed new ground-glass opacities and poorly defined centrilobular nodules. He had undergone catechin inhalation for 1 month. We diagnosed hypersensitivity pneumonitis (HP) based on the clinical course, bronchoscopy and a challenge test. Cases of HP due to inhalation of extracted catechin powder are rare. Although it has many known positive attributes, it is necessary to be aware that catechin can cause HP.

\section{KARGER}

Fax +4161306 1234

E-Mail karger@karger.ch

www.karger.com
C) 2011 S. Karger AG, Basel

0025-7931/11/0824-0388\$38.00/0

Accessible online at: www.karger.com/res

\section{Introduction}

Green tea is one of the most popular beverages in the world. Catechins are the major soluble component of dried Japanese green tea leaves. Recent studies have shown that tea catechins have hypocholesterolemic, antioxidative and antimutagenic effects [1]. Methicillin-resistant Staphylococcus aureus (MRSA) is a frequent cause of hospital-acquired pneumonia. Catechin inhalation appears to reduce the MRSA count in the sputum without significant side effects [2].

Hypersensitivity pneumonitis (HP) is a diffuse parenchymal lung disease characterized by immunologic reaction to various inhaled allergens including organic substances, some industrial chemical products and certain drugs. We describe here a patient who developed HP after inhalation of catechin-rich extracts. To the best of our knowledge, this type of case is very rare.

Hiroshi Otera, MD, $\mathrm{PhD}$

Department of Respiratory Medicine, Nishi-Kobe Medical Center

5-7-1 Kojidai, Nishi-ku

Kobe 651-2273 (Japan)

Tel. +81 78997 2200, E-Mail h.ohtera@nmc-kobe.org 

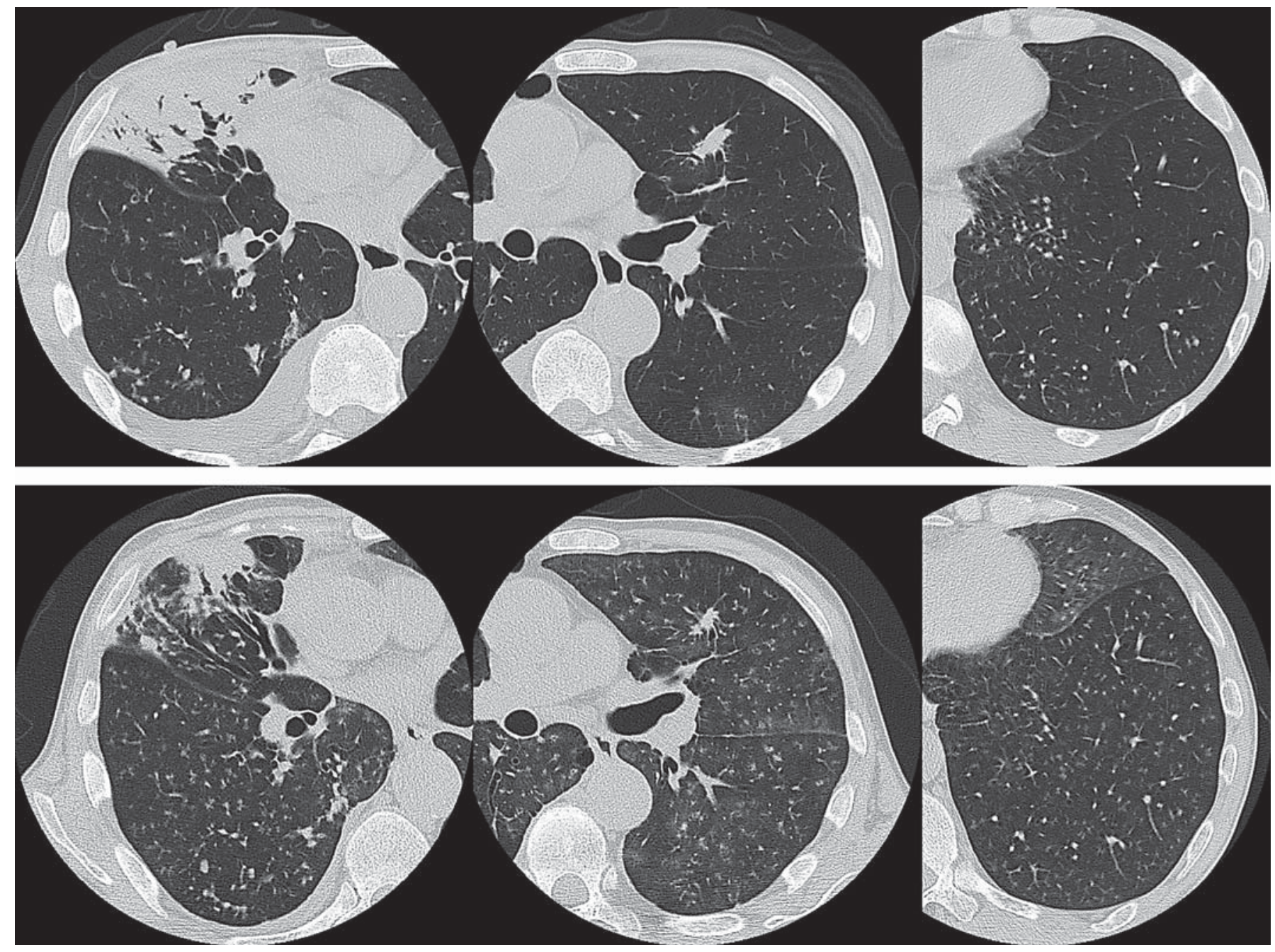

Fig. 1. HRCT images of the lung 3 months before the symptoms reported here (upper row) and at the time the symptoms were experienced (lower row). Centrilobular nodules accompanied by ground-glass opacities distributed in a geometric fashion (mosaic pattern) were observed in the images collected when the patient exhibited the symptoms. The right middle lobe consolidation, which we thought to be related to tuberculosis, improved after 3 months of treatment for tuberculosis.

\section{Case Report}

A 51-year-old man was admitted to our hospital with a diagnosis of tuberculosis. After 3.5 months of antituberculosis therapy, he suddenly experienced fever and general fatigue. His body temperature was $38.8^{\circ} \mathrm{C}$, heart rate 106 beats $/ \mathrm{min}$, blood pressure $124 / 72 \mathrm{~mm} \mathrm{Hg}$ and percutaneous oxygen saturation $88 \%$ in room air. Coarse crackles were audible over the posterior right lung. The white blood cell count was $9,500 / \mathrm{mm}^{3}$ with $15.1 \%$ eosinophils and $10.7 \%$ lymphocytes. The lactate dehydrogenase level was 301 IU/l, C-reactive protein $0.8 \mathrm{mg} / \mathrm{dl}$ and KL-6 2,320 U/ml. $\beta$-Dglucan was absent.

Chest radiography showed upper lung consolidation, scattered linear opacities and diffuse small nodules on both sides. High-resolution computed tomography (HRCT) of the chest re- vealed that extensive pleural-based consolidations and upper zone cavities on both sides were smaller than they were 3 months before. However, diffuse bilateral ground-glass opacities, poorly defined centrilobular nodules and a mosaic pattern of attenuation appeared (fig. 1). The patient underwent bronchoscopy with bronchoalveolar lavage and transbronchial biopsies. Cell differentiation of bronchoalveolar lavage fluid (BALF) showed $1.3 \times$ $10^{6}$ white blood cells $/ \mathrm{mm}^{3}$ (41.5\% eosinophils, $31.0 \%$ lymphocytes, $10.0 \%$ segmental and $10.0 \%$ macrophages), and his CD4/ CD8 ratio was 1.3. Transbronchial biopsy samples were taken from the right middle and lower segmental bronchus and, on microscopic examination, showed alveolitis with mainly lymphocytes (fig. 2). Ziehl-Neelsen staining for acid-fast bacilli was negative. The culture was negative for bacteria, fungi and mycobacteria. 


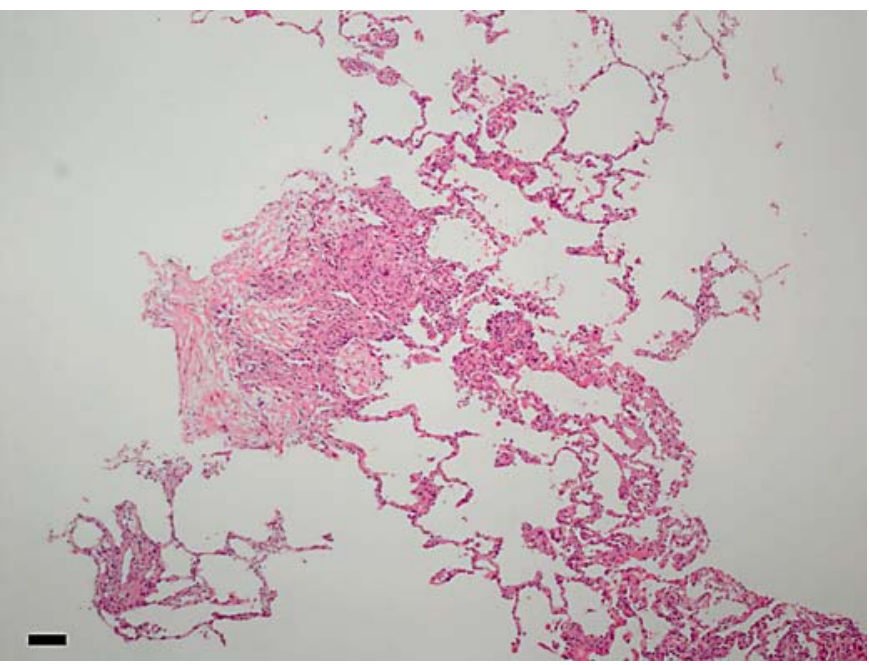

Fig. 2. Lung specimen obtained by transbronchial lung biopsy. Pathological findings revealed thickening of the alveolar walls by lymphocytic infiltration. There were no apparent granuloma formations or Masson bodies. Scale bar $=100 \mu \mathrm{m}$. Hematoxylin and eosin stain.

Table 1. Provocation test analysis

\begin{tabular}{|c|c|c|c|c|}
\hline & \multicolumn{4}{|c|}{ Hours after inhalation } \\
\hline & before & \multicolumn{3}{|l|}{4} \\
\hline \multicolumn{5}{|c|}{ Arterial blood gas analysis } \\
\hline $\mathrm{pH}$ & 7.388 & \multicolumn{2}{|c|}{7.444} & \\
\hline $\mathrm{pCO}_{2}, \mathrm{~mm} \mathrm{Hg}$ & 43.7 & \multicolumn{2}{|c|}{43.3} & \\
\hline $\mathrm{pO}_{2}, \mathrm{~mm} \mathrm{Hg}$ & 89.1 & \multicolumn{2}{|c|}{62.2} & \\
\hline $\mathrm{PaO}_{2} / \mathrm{FiO}_{2}$ ratio & 446 & \multicolumn{2}{|c|}{174} & \\
\hline \multirow[t]{3}{*}{ Oxygen therapy } & room ail & \multicolumn{3}{|c|}{ nasal prongs ( $4 \mathrm{l} / \mathrm{min})$} \\
\hline & \multicolumn{4}{|c|}{ Days after inhalation } \\
\hline & before & 1 & 2 & 5 \\
\hline \multicolumn{5}{|c|}{ Laboratory data analysis } \\
\hline $\mathrm{WBC}, / \mu \mathrm{l}$ & 6,600 & 19,200 & 10,200 & 6,600 \\
\hline Neutrophils, \% & 58.1 & 90.7 & & 59.4 \\
\hline Lymphocytes, \% & 20.2 & 5.0 & & 22.7 \\
\hline Eosinophils, \% & 8.5 & 1.6 & & 7.9 \\
\hline LDH, IU/l & 191 & 345 & 240 & 191 \\
\hline $\mathrm{CRP}, \mathrm{mg} / \mathrm{dl}$ & 0.1 & 6.3 & 4.5 & 0.6 \\
\hline
\end{tabular}

$\mathrm{LDH}=$ Lactate dehydrogenase $\mathrm{CRP}=$ C-reactive protein.
MRSA was detected in the patient's sputum at the time of the original hospitalization when tuberculosis was diagnosed. To decrease this, he had been treated with catechin inhalation for 1 month before the current episode. Inhalation of $2 \mathrm{ml}$ of catechinrich green tea extract solution (Teaflan 30A, Itoen Ltd., Tokyo, Japan) dissolved in distilled water (DW; $50 \mathrm{mg} / \mathrm{ml}$ ) was performed once or twice daily using a handheld nebulizer. Although the patient did not initially recognize any particular symptoms, he later noticed that he coughed frequently during inhalation of the extract and afterwards.

The patient had no history of allergic episodes. He had been orally administered isoniazid, rifampin and ethambutol every day after lunch. He had not left our ward, and a meticulous review of his environmental exposure showed no obvious external cause for HP. We discontinued the administration of antituberculosis drugs and inhalation and, 1 month later, the percentage of peripheral eosinophils decreased to $8.5 \%$.

We performed skin tests (patch skin test and scratch skin test) for catechin powder in DW solution at the same concentration used for inhalation. The results of both skin tests were negative. The results of drug lymphocyte stimulation tests for isoniazid, rifampin, ethambutol and catechin powder were also negative, so we performed an inhalation challenge test with only DW in the protocol described above. The symptoms did not change, and cell differentiation of induced sputum showed many lymphocytes and few neutrophils both before and after the DW provocation test. Next, we performed an inhalation challenge test using catechin. Four hours after inhalation, the patient experienced fever, dyspnea and productive cough. His body temperature was $39.8^{\circ} \mathrm{C}$, and percutaneous oxygen saturation was $88 \%$ in room air. Physical examination revealed coarse crackles, a flushed face and conjunctival injection. Blood examinations demonstrated remarkable changes (table 1). Six hours after inhalation, many neutrophils and few lymphocytes were present in his sputum.

We used clean devices for every inhalation. Bacterial and fungal cultures of the catechin solution showed negative results, and $\beta$-D-glucan was below detection levels. We restarted administration of antituberculosis drugs. The number of peripheral eosinophils increased again, but the patient's symptoms did not change, and the chest radiogram gradually cleared. These findings were consistent with HP due to inhalation of catechin-rich green tea extract solution.

\section{Discussion}

Catechins comprise about $15 \%$ by weight of dried Japanese green tea leaves and are the source of their astringent taste [1]. Catechins consist of several polyphenols: (-)-epigallocatechin gallate, epicatechin, gallocatechin, gallocatechin gallate, epigallocatechin and epicatechin gallate. Epigallocatechin gallate has been reported to be the causative agent for green tea-induced asthma [1]. The other components of green tea are caffeine, theanine, theaflavins, theobromine, theophylline and phenolic acids [3].

Recent studies have shown that green tea possesses many useful antibacterial, antioxidative, anti-inflamma- 
tory, antiallergic and antiarteriosclerotic properties [35]. Several side effects of green tea have been reported, including gastrointestinal complaints (abdominal bloating, constipation and nausea) and neurologic complaints (insomnia and restlessness) [4, 6], but most were not severe. There are some reports to suggest that green tea extracts may be related to liver damage [7]; however, green tea extract was categorized as Class A by the US Pharmacopeia based on later information [8]. Catechin inhalation has been reported to demonstrate an anti-MRSA effect [9]; it appeared to reduce the MRSA count in sputum and was safe [2]. For these reasons, we used catechin powder to treat patients with MRSA present in their sputum, even though this method is not routine.

$\mathrm{HP}$ is a diffuse parenchymal lung disease characterized by an allergic inflammatory reaction to a repeatedly inhaled allergen. Avian antigen and Mycobacterium avium complex are the most common of those antigens, and there have been many reports of HP caused by Trichosporon asahii and Trichosporon mucoides in Japan [10]. HP can be quite variable in its clinical presentation, severity and natural history. The clinical course is categorized as acute, subacute or chronic. The most common presenting symptoms are dyspnea and cough. HRCT is an essential clinical tool in the diagnosis of HP. Centrilobular micronodules with associated ground-glass opacities are a feature of HP, but this finding is not specific to HP. BALF of HP patients typically shows lymphocytosis. Histological findings in lung specimens differ according to HP stage. In the subacute course, bronchiolitis with lymphoplasmacytic infiltration, nonnecrotizing granulomas and Masson bodies are typical [11]. Various schemes of diagnostic criteria have been proposed for HP, but none have been universally accepted.

In our patient, the clinical history identified a causative antigen; moreover, clinical, radiographic and histological findings were consistent with HP, and the results of the challenge test were positive. Therefore, many clinicians would agree with our diagnosis of subacute HP. However, BALF analysis showed not only lymphocy tosis but also eosinophilic infiltration. BALF cell differentials may support the likelihood of a certain lung disease but are not specific for a particular disease [12]. Although several confounding factors could affect the interpretation of the results in our patient, one possible reason for this observation is the increased eosinophil count in the patient's peripheral blood, which was a side effect of the antituberculosis drugs. Upon histological examination, eosinophil infiltration was found to be low. After stopping antituberculosis medications for 1 month, the eo- sinophil count in the peripheral blood decreased and the sputum induced by the provocation test contained few eosinophils and many neutrophils.

Green tea-induced pulmonary adverse reactions are rare, and cases of green tea-induced asthma have been reported only rarely $[1,13]$. Moreover, only 1 case of catechin-induced HP has been previously reported. Sano et al. [14] described a 54-year-old woman with HP who had been making tea bags for 18 years. The HRCT findings and allergic skin test results were similar between this and the present case. However, the patients' reactions to provocation tests were very different. The earlier patient's symptoms and body temperature did not change after inhalation. Additionally, her white blood cell count was only elevated by $34 \% 6 \mathrm{~h}$ after inhalation and decreased to baseline $24 \mathrm{~h}$ after inhalation. Our patient's reaction to the provocation test was stronger and longer, and the time from inhalation to exhibition of symptoms was shorter. The provocation tests differed in that the previous study used a catechin concentration no higher than $1.0 \mathrm{mg} / \mathrm{ml}$, while we used a concentration of $50 \mathrm{mg} / \mathrm{ml}$. This difference may in part explain the stronger reaction we observed.

Thus, an unexpected cause may induce HP [15]. It is necessary to consider this entity with regard to the clinical course and radiological findings.

\section{Conclusion}

We present a case of HP induced by exposure to catechin powder, a previously unreported source of antigen in this syndrome. Catechin-induced lung disease is rare, and its low incidence may be attributable to failure to notice and identify the complication.

\section{Financial Disclosure and Conflicts of Interest}

None of the authors have any financial support or conflicts of interests to declare. 


\section{References}

$>1$ Shirai T, Sato A, Hara Y: Epigallocatechin gallate. The major causative agent of green tea-induced asthma. Chest 1994;106:18011805.

$>2$ Yamada H, Ohashi K, Atsumi T, Okabe H, Shimizu T, Nishio S, Li XD, Kosuge K, Watanabe $H$, Hara Y: Effects of tea catechin inhalation on methicillin-resistant Staphylococcus aureus in elderly patients in a hospital ward. J Hosp Infect 2003;53:229-231.

$>3$ Cooper R, Morré DJ, Morré DM: Medicinal benefits of green tea. Part I. Review of noncancer health benefits. J Altern Complement Med 2005; 11:521-528.

4 Cooper R, Morré DJ, Morré DM: Medicinal benefits of green tea. Part II. Review of anticancer properties. J Altern Complement Med 2005;11:639-652.

$>5$ Koo MW, Cho CH: Pharmacological effects of green tea on the gastrointestinal system. Eur J Pharmacol 2004;500:177-185.
6 Pisters KM, Newman RA, Coldman B, Shin DM, Khuri FR, Hong WK, Glisson BS, Lee JS: Phase I trial of oral green tea extract in adult patients with solid tumors. J Clin Oncol 2001;19:1830-1838.

7 Sarma DN, Barrett ML, Chavez ML, Gardiner P, Ko R, Mahady GB, Marles RJ, Pellicore LS, Giancaspro GI, Low Dog T: Safety of green tea extracts: a systematic review by the US Pharmacopeia. Drug Saf 2008;31:469484.

8 Update on the USP Green Tea Extract Monograph. US Pharmacopeia. Posted 10 April 2009. http://www.usp.org/USPNF/notices/ updateUSPGreenTeaExtractMonograph. html.

-9 Stapleton PD, Shah S, Ehlert K, Hara Y, Taylor PW: The $\beta$-lactam-resistance modifier (-)-epicatechin gallate alters the architecture of the cell wall of Staphylococcus aureus. Microbiology 2007;153:2093-2103.

10 Hanak V, Golbin JM, Ryu JH: Causes and presenting features in 85 consecutive patients with hypersensitivity pneumonitis. Mayo Clin Proc 2007;82:812-816.
1 Barrios RJ: Hypersensitivity pneumonitis. Arch Pathol Lab Med 2008;132:199-203.

12 Welker L, Jörres RA, Costabel U, Magnussen $\mathrm{H}$ : Predictive value of BAL cell differentials in the diagnosis of interstitial lung diseases. Eur Respir J 2004;24:1000-1006.

13 Shirai T, Reshad K, Yoshitomi A, Chiba K, Nakamura H, Taniguchi M: Green tea-induced asthma: relationship between immunological reactivity, specific and non-specific bronchial responsiveness. Clin Exp Allergy 2003;33:1252-1255.

14 Sano T, Gemma H, Satou M, Ono T, Atsumi E, Ito I, Chida K, Suda T, Nakamura H, Toyoshima M, Shirai T: A case of hypersensitivity pneumonitis in a tea bag manufacturer employee (in Japanese). Nihon Kyobu Rinsho 2004;63:393-398.

15 Tonelli AR, Lottenberg R, Allan RW, Sriram PS: Rituximab-induced hypersensitivity pneumonitis. Respiration 2009;78:225-229. 